\title{
Impacto de uma intervenção educacional de enfermagem em pacientes portadores de DPOC
}

\author{
Impact of an educational nursing intervention in patients with COPD \\ Impacto de una intervención educativa de enfermería en pacientes con EPOC
}

\author{
Renata Camargo Alves'; Luciane Soares de Limall; Dulce Aparecida Barbosa"II; \\ Silvana Andrea Molina Lima ${ }^{\text {IV }}$; Ana Rita de Cássia Bettencourt ${ }^{V}$
}

\begin{abstract}
RESUMO
Objetivo: avaliar os efeitos imediatos da orientação do plano de alta hospitalar aos pacientes com DPOC. Método: estudo prospectivo, comparativo, realizado num hospital privado de São Paulo, com pacientes com DPOC. Na primeira etapa foi aplicado questionário "Plano educacional para pacientes com DPOC" para avaliar conhecimento do paciente sobre sua doença. Na segunda etapa foi realizada intervenção educacional e na terceira, reaplicação do questionário. A comparação do resultado do pré e pós-teste foram feitas pelo teste $\mathrm{T}$ pareado. Projeto de pesquisa aprovado em comitê de ética, parecer $\mathrm{n}^{\circ} 003 / 12$. Resultados: dos 50 pacientes participantes do estudo, $68 \%$ responderam não conhecer bem sua doença antes da orientação $e$ apenas $22 \%$ após intervenção educacional. Todas seções do questionário apresentaram aumento na média de acertos após intervenção educacional. Conclusão: orientação do plano de alta hospitalar mostrou-se efetiva resultando em melhora imediata do conhecimento.
\end{abstract}

Descritores: Alta do paciente; doença pulmonar obstrutiva crônica; educação em enfermagem; educação em saúde.

\begin{abstract}
Objective: to evaluate the immediate effects of the orientation of the hospital discharge plan for patients with COPD. Method: prospective and comparative study performed in a private hospital in São Paulo, with patients with COPD. In first step it was used the questionnaire "Educational Plan for COPD patients" to assess patient knowledge about their disease. In second step, educational intervention was carried out and in the third, again the of the questionnaire was used. The comparison between pre and post-test results was done by paired T-test. The project was approved by the research ethics committee. Result: from the 50 patients who participated in study, $68 \%$ reported not knowing their disease well before orientation and only $22 \%$ after educational intervention. In all sections of questionnaire there was an increase in correct answers after educational intervention. Conclusion: the orientation of the hospital discharge plan was effective resulting in an immediate improvement of knowledge.

Descriptors: Patient discharge; pulmonary disease, chronic obstructive; education, nursing; health education.

\section{RESUMEN}

Objetivo: evaluar los efectos inmediatos de la orientación del plan de alta para pacientes con EPOC. Métodos: estudio prospectivo, comparativo, realizado en hospital privado de São Paulo, con pacientes con EPOC. En la primera etapa se aplicó el cuestionario "Plan de Educación para los pacientes con EPOC" para evaluar el conocimiento del paciente sobre su enfermedad. En la segunda etapa fué realizada intervención educativa, en la tercera se realizó reaplicación del cuestionario. La comparación del resultado del pre y post-test fue hecha por la prueba T pareado. Proyecto de investigación aprobado por comité de ética, protocolo $n^{\circ} 003 / 12$. Resultados: de los 50 pacientes participantes, $68 \%$ dijeron desconocer su enfermedad antes de la orientación, sólo $22 \%$ después de la intervención educativa. Todas secciones del cuestionario se produjo aumento en promedio de aciertos después de la intervención. Conclusión: la orientación del plan de alta fué eficaz, resultando mejora inmediata del conocimiento.
\end{abstract}

Descriptores: Alta del paciente; enfermedad pulmonar obstructiva crónica; educación en enfermería; educación en salud.

\section{INTRODUÇÃO}

As doenças crônicas representam um considerável problema para a sociedade devido à alta mortalidade e sobrecarga econômica durante o tratamento, além de representar um desafio para as equipes de saúde no que diz respeito à sensibilização dos pacientes durante seu preparo para a alta a fim de atingir adesão ao tratamento.

\footnotetext{
'Enfermeira. Aluna de Doutorado do Programa de Pós-Graduação em Enfermagem da Faculdade de Medicina de Botucatu - Unesp. São Paulo, Brasil. E-mail: recamargoalves@gmail.com

"Enfermeira. Pós Doutorado em Enfermagem. Professora Titular do Departamento de Enfermagem. Universidade Federal de Pernambuco. Pernambuco, Brasil. E-mail: luciane.lima@globo.com

II'Enfermeira. Pós Doutorado em Nefrologia. Professora Associada IV - Livre Docente da Escola Paulista de Enfermagem. Universidade Federal de São Paulo. São Paulo, Brasil. E-mail: dulce.barbosa@unifesp.br

IVEnfermeira. Professora Assistente Doutora do Departamento de Enfermagem da Faculdade de Medicina de Botucatu - Unesp. São Paulo, Brasil. E-mail: smolina@fmb.unesp.br

VEnfermeira. Doutora em Enfermagem. Professora Adjunta da Escola Paulista de Enfermagem. Universidade Federal de São Paulo. São Paulo, Brasil. E-mail: arcbettencourt@unifesp.br
} 
A doença pulmonar obstrutiva crônica (DPOC) é uma doença com evolução progressiva e crônica. Seu tratamento visa principalmente melhorar a qualidade de vida do paciente e diminuir a progressão do processo patológico. Apesar dos entraves encontrados pela equipe assistencial e do emprego do modelo biomédico nos sistemas de saúde, vemos um aumento dos investimentos para que o paciente se aproprie de conhecimentos para realizar o manejo de sua doença de maneira eficaz e segura.

Sendo assim, mensurar evidências que nos façam refletir sobre os efeitos e repercussões da orientação aos pacientes com quadro de DPOC podem nos trazer discussões acerca do processo de tratamento, do grau de conhecimento desses sujeitos antes e após a orientação de alta de enfermagem e da oportunidade que o profissional de enfermagem tem disponível para educar e sensibilizar os indivíduos sobre a sua patologia e, portanto, contribuir para a atuação da equipe e proporcionar ao paciente conhecimento sobre seu quadro e melhores condições de autocuidado após a alta.

Este estudo teve como objetivo avaliar os efeitos imediatos da orientação do plano de alta hospitalar aos pacientes portadores de DPOC acerca de sua doença.

\section{REVISÃO DE LITERATURA}

A DPOC é uma enfermidade respiratória com manifestações sistêmicas, caracterizada pelo desenvolvimento progressivo de limitação ao fluxo aéreo que não é totalmente reversível. A dispneia pode ser intensa e com frequência interfere nas atividades diárias do paciente ${ }^{1}$.

A DPOC gera substancial sobrecarga econômica sobre o indivíduo portador da doença e sobre o sistema de saúde, devido a redução de atividade e internações hospitalares ${ }^{2,3}$.

O tratamento da doença visa principalmente melhorar a qualidade de vida do paciente e diminuir a progressão do processo patológico. Durante a internação hospitalar, o foco do atendimento é voltado para tratar as vias aéreas obstruídas, através do uso de broncodilatadores, antibióticos, oxigenioterapia, nutrição adequada, fisioterapia respiratória e apoio psicológico ${ }^{4-6}$. Por essas razões, essa doença tem sido motivo de numerosos estudos, principalmente nos assuntos referentes ao processo educativo do paciente.

No Brasil, a educação dos pacientes é realizada de maneira ainda discreta, resultando em elevado número de reinternações hospitalares. Devido à sobrecarga de tarefas e inúmeras atribuições, o enfermeiro realiza a orientação de alta de forma protocolar, não levando em conta as condições específicas de cada paciente ${ }^{7}$.

O planejamento da alta deve ser realizado por uma equipe interdisciplinar tendo o enfermeiro como o responsável pelo elo entre os profissionais. O plano de alta consiste em uma forma organizada de exprimir as atividades determinadas pelas particularidades de cada paciente, sendo que o ideal seria que seu planejamento fosse iniciado logo após o momento da admissão, para que o enfermeiro tenha a possibilidade de esclarecer dúvidas e avaliar os resultados efetivos no conhecimento ${ }^{8}$.

O enfermeiro deve elaborar um plano de alta compatível com o grau de compreensão do paciente e orientá-lo a fim de lhe transmitir conhecimentos que possam modificar o seu comportamento de saúde, principalmente quando se trata de doenças crônicas. Portanto, é fundamental que os profissionais de saúde entendam como a DPOC afeta os pacientes não apenas do ponto de vista fisiológico, mas também, o lado psicológico e social para que as condutas de enfermagem sejam efetivas e para que o plano de intervenções elaborado seja coerente com as suas expectativas e possibilidades ${ }^{9,10}$.

Nesse sentido, estudos relacionados à educação em saúde são fundamentais para contribuir com a atuação da equipe e proporcionar ao paciente conhecimento sobre seu quadro e melhores condições de autocuidado após a alta, a fim de realizar o manejo de sua doença de maneira eficaz e segura diminuindo as complicações e internações hospitalares ${ }^{11,12}$.

Este estudo tem como objetivo avaliar os efeitos imediatos da orientação do plano de alta hospitalar aos pacientes portadores de DPOC.

\section{MÉTODO}

Estudo prospectivo, comparativo, do tipo antes e depois, realizado nas unidades de internação de um hospital de caráter privado, de grande porte, localizado na cidade de São Paulo.

A coleta de dados foi realizada no período de maio de 2012 a maio de 2013 e a amostra foi composta por 50 pacientes que aceitaram participar do estudo. Não houve recusa ou desistência durante a pesquisa. Foram incluídos no estudo pacientes com diagnóstico de DPOC segundo o prontuário de internação e que permaneceram internados por um período superior a três dias. Foram excluídos os pacientes que apresentaram pontuação no Mini Exame do Estado 
Mental (MEEM) abaixo de 18 (para pacientes sem instrução formal) e abaixo de 24 (com pelo menos 1 ano de instrução escolar). Este instrumento visa avaliar a capacidade cognitiva do paciente e é composto por sete categorias: orientação temporal ( 0 a 5 pontos), orientação espacial ( 0 a 5 pontos), registro de três palavras ( 0 a 3 pontos), atenção e cálculo (0 a 5 pontos), memória recente ( 0 a 3 pontos), linguagem ( 0 a 8 pontos) e capacidade construtiva visual ( 0 a 1 ponto). Sua pontuação pode variar de 0 a 30 pontos $^{13}$.

O questionário utilizado para avaliar o conhecimento do paciente sobre sua doença foi o Plano Educacional para pacientes com doença pulmonar obstrutiva crônica, instrumento de avaliação do conhecimento, desenvolvido e validado pelo Centro de Reabilitação Pulmonar da Universidade Federal de São Paulo/Lar Escola São Francisco (UNIFESP/LESF), na cidade de São Paulo. Composto por 30 questões e dividido em sete seções que abordam respectivamente: anatomia do sistema respiratório; atividade diária; mobilização de secreção; prática de exercício físico; uso de medicação; fumo e alimentação. A questão 30 aborda a percepção do paciente sobre o autoconhecimento de sua doença. Cada questão possui quatro alternativas, sendo que a última alternativa tem como resposta: não sei; exceto a questão de número 30 que possui duas alternativas (sim ou não). A pontuação deste questionário é dada pela porcentagem de acertos e erros; sendo possível saber o grau de informação que o paciente possui sobre a sua doença ${ }^{14}$.

Diariamente a pesquisadora acessou o software de gestão de saúde da instituição hospitalar que possibilitava o acesso à lista dos pacientes internados nas unidades de internação e seus respectivos diagnósticos.

$\mathrm{O}$ estudo foi realizado em três etapas, em três dias consecutivos. No primeiro dia, a pesquisadora aplicava o formulário contendo as variáveis sociodemográficas e clínicas, o MEEM e o questionário "Plano Educacional para pacientes com doença pulmonar obstrutiva crônica" para avaliar o conhecimento sobre a patologia e tratamento da DPOC, denominado como pré-teste.

Toda a abordagem foi realizada no apartamento hospitalar com ou sem a presença de acompanhante. Nos casos em que o acompanhante estava presente, estes eram orientados pela pesquisadora a não interferirem na resposta do paciente e que todas as suas dúvidas seriam esclarecidas ao término do estudo.

No segundo dia foi realizado um trabalho de intervenção educativa de modo a dialogar com o paciente sobre as sete seções do questionário "Plano Educacional para pacientes com doença pulmonar obstrutiva crônica" com duração média de 1 hora e meia. Toda a orientação era feita com auxílio de material educativo ilustrado e impresso que era entregue ao paciente ao final do estudo. No terceiro dia de abordagem, foi aplicado novamente o questionário "Plano Educacional para pacientes com doença pulmonar obstrutiva crônica”, denominado no estudo como pós-teste. Ao término das respostas, era aberto novamente um espaço para que o paciente e acompanhante esclarecessem suas dúvidas e era entregue o material educativo. Este material foi elaborado pela pesquisadora tomando-se por base todo o conteúdo que seria abordado.

Os dados categóricos foram resumidos através da frequência absoluta e relativa ( $\mathrm{n}$ e \%) e média, desvio padrão mínimo e máximo, no caso de variáveis numéricas. A comparação do resultado do pré e pós-teste foram feitas pelo teste T pareado, através da comparação do número de respostas corretas no total e por seção. A comparação entre o pré e pós-teste acerca do conhecimento da doença foi realizada pelo teste de Mc Nemar (significância das mudanças). A significância estatística foi obtida para valores de $p<0,05$.

O estudo obteve aprovação do Comitê de Ética em Pesquisa do Hospital Nove de Julho (CEPH9J) Parecer no 003/12, tendo como coparticipante a Universidade Federal de São Paulo (CEP UNIFESP) Parecer no 777.980. Os participantes assinaram o Termo de Consentimento Livre e Esclarecido.

\section{RESULTADOS}

Participaram do estudo 50 pacientes portadores de DPOC, sendo $50 \%$ do gênero masculino, a idade média foi de $73,5$ anos (DP $\pm 10,8)$. A média de escolaridade foi de 12,8 anos $(\mathrm{DP} \pm 4,018)$ e a média do MEEM foi de 26,8 pontos $(\mathrm{DP} \pm 1,8)$.

Em relação aos dados sobre tabagismo, 76\% eram ex-tabagistas, 14\% tabagistas e $10 \%$ nunca fumou. 0 tempo médio de fumo foi de 38,2 anos $(D P \pm 14,7)$ e o consumo de cigarro, medido em anos/maço, foi de 55,0 (DP $\pm 45,2)$. 0 tempo médio do diagnóstico de DPOC foi de 5,6 anos (DP $\pm 6,9)$.

Em relação à gravidade da DPOC, $4 \%$ da amostra encontravam-se no grau I (DPOC leve), 58\% no grau II (DPOC moderada), $22 \%$ no grau III (DPOC grave) e 16\% no grau IV (DPOC muito grave).

A maioria dos pacientes (84\%) realizava tratamento para DPOC e 78\% com regularidade. Quanto à medicação, $50 \%$ faziam uso de broncodilatador inalatório e $42 \%$ de corticoide inalatório.

A maioria dos pacientes não procuravam informações sobre a doença (74\%). Dentre os que mostravam interesse, $12 \%$ realizavam esta busca em consultório médico, $8 \%$ na internet, $4 \%$ em livros e $2 \%$ através de outros meios. 
A tabela 1 traz a média de acertos e o desvio-padrão no pré e pós-teste das sete seções do questionário, bem como a média da diferença entre os resultados do pré e pós-teste por seção e os seus respectivos desvio-padrão. Em todas as seções houve um aumento na média do pós-teste comparada à média de acertos no pré-teste. A análise estatística mostra que houve aumento na média do pós-teste comparada à média de acertos no pré-teste em todas as seções $(p<0,0001)$. Os dados mostram que as seções 1 e 5 que abordavam respectivamente, a anatomia do sistema respiratório e o uso de medicação foram as que apresentaram maior diferença na média de acertos, já a seção 6 que abordou o fumo, foi a que apresentou a menor diferença.

Tabela 1: Variação da média e desvio padrão do número de acertos - São Paulo - maio de 2012 a maio de 2013

\begin{tabular}{|c|c|c|c|c|}
\hline Seções do questionário & $\begin{array}{l}\text { Pré-teste } \\
\text { média } \\
\left(\mathrm{DP}^{*}\right)\end{array}$ & $\begin{array}{l}\text { Pós-teste } \\
\text { média } \\
\left(D^{*}\right)\end{array}$ & $\begin{array}{l}\text { Diferença entre } \\
\text { os resultados do } \\
\text { pré e pós-teste }\end{array}$ & $\begin{array}{l}\text { p-value }{ }^{\dagger} \text { Diferença } \\
\text { entre os resultados } \\
\text { do pré e pós-teste }\end{array}$ \\
\hline Seção 1 - Anatomia do sistema respiratório (5 questões) & $2,7(0,99)$ & $4,4(0,83)$ & $-1,7(0,87)$ & $<0,0001+$ \\
\hline Seção 2 - Atividades diárias (3 questões) & $1,9(0,95)$ & $2,6(0,67)$ & $-0,7(0,83)$ & $<0,0001+$ \\
\hline Seção 3 - Mobilização de secreção (3 questões) & $1,9(0,77)$ & $2,6(0,54)$ & $-0,7(0,76)$ & $<0,0001+$ \\
\hline Seção 4 - Práticas de exercícios físicos (4 questões) & $2,5(0,76)$ & $3,4(0,63)$ & $-0,8(0,79)$ & $<0,0001+$ \\
\hline Seção 5 - Uso de medicação (4 questões) & $1,9(1,33)$ & $3,7(0,59)$ & $-1,7(1,18)$ & $<0,0001+$ \\
\hline Seção 6 - Fumo (5 questões) & $4,2(0,76)$ & $4,6(0,54)$ & $-0,4(0,63)$ & $<0,0001+$ \\
\hline Seção 7 - Alimentação (5 questões) & $3,0(1,17)$ & $3,7(0,89)$ & $-0,7(1,07)$ & $<0,0001+$ \\
\hline
\end{tabular}

*DP - desvio padrão; † diferença estatisticamente significativa

Em relação à última questão, que abordava a percepção sobre o autoconhecimento da doença, as porcentagens de respostas positivas e negativas no pré e pós-teste estão dispostas na tabela 2.

Tabela 2: Porcentagem de respostas positivas e negativas no pré e pós-teste acerca da percepção sobre seu autoconhecimento da doença - São Paulo - maio de 2012 a maio de 2013.

\begin{tabular}{lcc}
\hline Percepção sobre o autoconhecimento da doença & Sim & Não \\
& (\%) & (\%) \\
\hline Pré-teste & 32 & 68 \\
Pós-teste & 88 & 12 \\
\hline
\end{tabular}

No pré-teste houve predominância na porcentagem de pacientes com respostas negativas (68\%), já no pós-teste, após a orientação, houve predomínio na porcentagem de pacientes que responderam "sim" (88\%), afirmando conhecer sua doença.

\section{DISCUSSÃo}

Os resultados deste estudo em relação à proporção de homens e mulheres acompanham a tendência observada em outros estudos. Atualmente, temos uma equivalência entre pacientes do sexo feminino e masculino portadores de DPOC. Este fato pode ser justificado pelo aumento do consumo do tabaco entre as mulheres ${ }^{2}$. A média da idade da amostra também está compatível com outros estudos, observando-se o predomínio de pacientes idosos ${ }^{2,15}$. Estudo de base populacional realizado no munícipio de São Paulo evidenciou que a prevalência da DPOC está associada com idade acima de 60 anos $^{2}$.

Em relação à capacidade cognitiva, a média do MEEM foi de 26,8 pontos, valor que representa uma boa capacidade cognitiva. Resultados diferentes foram encontrados no estudo chinês realizado para avaliar as alterações no hipocampo e o déficit cognitivo na DPOC, em que afirma que a hipoxemia crônica leva a uma atrofia hipocampal avaliada por ressonância magnética. Nesse estudo, a média apresentada foi de 28 para o grupo controle (indivíduos sadios), 24,57 para DPOC leve a moderada e 22,15 para DPOC grave $^{16}$. 
O predomínio de ex-tabagistas é similar ao encontrado em outros estudos ${ }^{2,17}$. Os dados sobre tabagismo como tempo médio de fumo e consumo de cigarro e o tempo médio de DPOC também está em consonância com outros estudos $^{2,17}$.

Quanto à gravidade da DPOC, a maioria foi classificada como grau II (58\%) e grau III (22\%), ou seja, DPOC moderada e grave respectivamente, similar ao encontrado na literatura. Esse fato talvez possa ser explicado pelas pesquisas terem sido realizadas em ambiente hospitalar e os pacientes que mais apresentam exacerbações e complicações são aqueles que estão nos estágios mais avançados da doença ${ }^{17,18}$.

A maioria dos pacientes (84\%) relatou realizar tratamento para doença e $78 \%$ referiu que o fazia com regularidade. Em relação ao uso de medicamento, metade da amostra fazia uso de broncodilatador inalatório e $42 \%$ de corticoide inalatório. Estudo espanhol realizado com 195 pacientes portadores de DPOC mostrou que a adesão ao tratamento tende a mudar ao longo do tempo. A adesão foi mensurada através da contagem de dose utilizada/dose prescrita. De acordo com os dados apresentados, na primeira visita o índice foi de 68,1\%, na segunda visita de $80 \%$ e na terceira, 84\%. Estes dados nos mostram a importância da orientação e acompanhamento dos pacientes pelos profissionais de saúde $^{19}$. Pesquisa realizada em Taiwan avaliou a técnica inalatória de 298 pacientes com DPOC e mostrou que a taxa de uso indevido é bastante alta e está diretamente relacionada ao conhecimento incorreto sobre a utilização do inalador, enfatizando a necessidade da educação sobre a terapia respiratória ${ }^{20}$. Estudo francês avaliou o manuseio do dispositivo inalatório em 2935 pacientes com DPOC e evidenciou erros em mais de 50\% das manipulações, independentemente do dispositivo utilizado, além disso, associou erros críticos à ocorrência de exacerbações graves da DPOC. Resultados da pesquisa comprovaram que o treinamento sobre o uso de inalador é parte integrante do gerenciamento da doença ${ }^{21}$.

Em relação ao questionário, observou-se que a seção 1 apresentou uma maior diferença na média de acertos entre o pré e pós-teste $(-1,7)$. Justifica-se este fato, pela seção conter questões muito específicas sobre anatomia do sistema respiratório. Entretanto, apesar das especificidades das questões, todas eram necessárias para proporcionar o conhecimento da doença como um todo.

A seção 2, sobre atividades diárias, apresentou um aumento discreto $(-0,7)$, porém significativo no número de acertos após a intervenção educacional. Estudo brasileiro realizado com 40 pacientes portadores de DPOC mostrou que estes são menos ativos que idosos saudáveis e passam a maior parte de seu tempo deitados ou sentados ${ }^{22}$. Entretanto, é fundamental que estas limitações funcionais sejam abordadas e as técnicas de conservação de energia ensinadas para que o paciente obtenha maior funcionalidade. Pesquisa brasileira mostrou que a prevalência de sintomas depressivos em pacientes com DPOC é alta e está diretamente relacionada ao comprometimento para realizar atividades de vida diária ${ }^{23}$. Estudo realizado na China avaliou a eficácia, antes e 12 meses após a realização e acompanhamento de um programa de reabilitação pulmonar em pacientes que receberam alta hospitalar após exacerbação da DPOC. Os 267 pacientes que completaram o programa de estudo apresentaram redução significativa nos episódios de exacerbação, nas visitas aos serviços de emergência e em episódios de hospitalização. Os resultados desta pesquisa foram atribuídos à técnica da respiração com lábios franzidos e a fisioterapia respiratória que resultaram no aumento da coordenação das funções neuromusculares e eficiência respiratória ${ }^{24}$.

A mobilização de secreção abordada na seção 3 também apresentou uma média de diferença entre o pré e pósteste de -0,7. De acordo com um estudo realizado na França, a tosse crônica e a produção de expectoração estão associadas com exacerbações de DPOC que muitas vezes requer hospitalização do paciente ${ }^{25}$. Por este motivo, é fundamental que o paciente saiba reconhecer alterações na quantidade e qualidade da tosse e expectoração.

A inatividade física está associada à exacerbação do quadro resultando em hospitalização. É essencial que a prática de exercícios físicos faça parte de um programa educativo para pacientes com DPOC, visto que aumentar os níveis de atividade é crucial para gestão da doença e pode resultar em melhora do prognóstico em longo prazo ${ }^{26}$. Em relação à seção 4 que abordou a prática de exercícios físicos, a média da diferença foi de $-0,8$, demonstrando que os pacientes entenderam a importância da mudança de hábito para a melhora da saúde.

Assim como a seção 1, a seção 5 que abordava o uso de medicação também apresentou uma maior diferença na média de acertos entre o pré e pós-teste $(-1,7)$. Estes dados estão em consonância com estudo voltado para construção de um plano de ensino que enfatiza a necessidade de o paciente aprender sobre os efeitos dos medicamentos e como utilizá-lo, além de revelar que pacientes adultos mais velhos geralmente apresentam maior dificuldade em utilizar inaladores e nebulizadores ${ }^{27}$.

A menor diferença na média de acertos entre o pré e o pós-teste $(-0,4)$ foi em relação ao fumo. Apesar da ampla divulgação pela mídia dos malefícios causados pelo cigarro, é necessário abordar esse assunto continuamente em programas educativos, a fim de sensibilizar o paciente. A cessação do tabagismo deve ser tratada como prioridade já que retarda a progressão da doença e aumenta a qualidade de vida ${ }^{28}$. 
Quanto à alimentação, a média da diferença foi de -0,7. A orientação nutricional é fundamental para DPOC, já que pacientes desnutridos apresentam maior aprisionamento de gás e redução no desempenho durante a prática de exercícios físicos ${ }^{28}$. Estudo espanhol evidenciou que pacientes com DPOC desnutridos apresentam piores níveis de funcionalidade do que os que com estado nutricional normal ${ }^{29}$.

Na questão 30 , que abordava a percepção sobre o autoconhecimento da doença, $68 \%$ dos pacientes responderam não conhecer bem sua doença antes da orientação e $22 \%$ após a intervenção educacional. De acordo com estudo realizado para avaliar percepções dos usuários com doenças crônicas, é fundamental que o profissional utilize estratégias com o objetivo de tornar o paciente seu parceiro na produção e informação de cuidados, transformando-o no gerenciador de sua própria doença. Para isto, é necessário permitir que o paciente adquira uma consciência crítica sobre seus problemas de saúde, tornando-o assim, mais seguro quanto à evolução e prognóstico de sua doença ${ }^{30}$.

O processo educativo do paciente e familiar durante a internação hospitalar ganhou destaque nos últimos tempos e tem apresentado resultados relevantes principalmente para doenças crônicas ${ }^{31}$. Vale destacar que a participação do enfermeiro neste processo é fundamental para se atingir os resultados esperados ${ }^{32}$.

Este estudo apresenta limitações visto que foi voltado para avaliar o conhecimento imediato adquirido. Para demonstrar se esta metodologia modificou o conhecimento tardio e interferiu no número de reinternações destes pacientes, teríamos que prolongar o tempo de coleta de dados por um período demasiadamente longo.

\section{CONCLUSÃO}

Os resultados deste estudo demonstraram que a intervenção educacional de enfermagem apresentou um impacto positivo sobre o aprendizado do paciente, resultando no aumento do seu conhecimento em todas as seções abordadas no questionário, além de uma melhora na autopercepção em relação a sua doença. 0 emprego do material ilustrativo utilizado no plano de alta hospitalar foi bem recebido e auxiliou no processo educativo. Estes resultados podem contribuir para o planejamento da orientação de alta hospitalar aos pacientes portadores de doenças crônicodegenerativas em geral, visando à melhora da qualidade de vida e de saúde.

\section{REFERÊNCIAS}

1. Bagatin E, Jardim JRB, Stirbulov R. Occupational chronic obstructive pulmonary disease. J Bras Pneumol. 2006 [cited 2017 Aug 25]; 32 Suppl 2: S35-40. DOI: http://dx.doi.org/10.1590/S1806-37132006000800007.

2. Sousa CA, César CLG, Barros MBA, Carandina L, Goldbaum M, Pereira JCR. Prevalence of chronic obstructive pulmonary disease and risk factors in São Paulo, Brazil, 2008-2009. Rev Saúde Pública. 2011 [cited 2017 Aug 25]; 45(5):887-96. DOI: http://dx.doi.org/10.1590/S0034-89102011005000051.

3. Örnek T, Tor M, Altın R, Atalay F, Geredeli E, Soyul O, et al. Clinical Factors Affecting the Direct Cost of Patients Hospitalized with Acute Exacerbation of Chronic Obstructive Pulmonary Disease. Int J Med Sci. 2012 [cited 2017 Aug 25]; 9(4): 285-290. DOI: http://dx.doi.org/10.7150/ijms.4039.

4. Cardoso AP. Exacerbations of COPD. Pulmão RJ. 2013 [cited 2017 Aug 25]; 22(2):60-64. Available from: http://www.sopterj.com.br/wp-content/themes/_sopterj_redesign_2017/_revista/2013/n_02/13.pdf.

5. Wedzicha JA; Decramer M; Seemungal TA. The role of bronchodilator in the prevention of exacerbations of COPD. Eur Respir J. 2012 [cited 2017 Aug 22]; 40(6): 1545-54. DOI: http://dx.doi.org/10.1183/09031936.00048912.

6. Machado DC, Camilo GB, Noronha AJ, Montessi OVD, Capone R, Capone D. Radiological diagnosis of COPD. Pulmão RJ 2013 [cited 2017 Aug 22]; 22(2):45-49. Available from: http://www.sopterj.com.br/wpcontent/themes/_sopterj_redesign_2017/_revista/2013/n_02/10.pdf.

7. Camargo PF, André LD, Lamari NM. Guidelines in health regarding hospital discharge process in readmitted users in the united health system. Arq Ciênc Saúde. 2016 [cited 2017 Aug 22]; 23(3) 38-43. Available from: https://pdfs.semanticscholar.org/b5dc/c13b6d55104500fc1b47a78c797d1673c8ac.pdf.

8. Santos I, Sarat CNF. Application of Orem's self-care theory in Brazilian scientific communications on nursing. Rev enferm UERJ. 2008 [cited 2017 Aug 25]; 16(3):313-18. Available from: http://www.facenf.uerj.br/v16n3/v16n3a03.pdf.

9. Hopkinson NS, Englebretsen C, Cooley N, Kennie K, Lim M, Woodcock T, et al. Designing and implementing a COPD discharge care bundle. Thorax. 2012 [cited 2017 Aug 25]; 67(1): 90-92. DOI: http://dx.doi.org/10.1136/thoraxjnl-2011-200233.

10. Veronez M, Higarashi IH. A protocol for hospital discharge of premature babies: input to building a proposal. Rev enferm UERJ. 2016 [cited 2017 Aug 25]; 24(3):e7505. DOI: http://dx.doi.org/10.12957/reuerj.2016.7505.

11. Silva RVGOS, Ramos FRS. The process of releasing a child from the hospital: nursing perceptions concerning limits and potentialities of its part in integral care. Texto Contexto Enferm. 2011 [cited 2017 Aug 22]; 20(2): 247-54. DOI: http://dx.doi.org/10.1590/S0104-07072011000200005.

12. Barbosa L, Borges PCP, Lemos SS, Cesarino CB. Evaluation of group educational intervention for diabetics receiving care at Teaching Clinic. Rev enferm UERJ. 2016 [cited 2017 Aug 25]; 24(2): e4968. DOI: http://dx.doi.org/10.12957/reuerj.2016.4968.

13. Chaves MLF [página na internet]. Testes de avaliação cognitiva: Mini-Exame do Estado Mental. [cited 2017 Aug 20]. Available from: http://www.cadastro.abneuro.org/site/arquivos_cont/8.pdf. 
14. Araújo PD. Plano educacional para pacientes com doença pulmonar obstrutiva crônica: Validação de um questionário [dissertação]. São Paulo (SP): UNIFESP; 2001 [cited 2017 Aug 25]. Available from: http://repositorio.unifesp.br/handle/11600/17135.

15. Qian J, Wastila LS, Rattinger GB, Zuckerman IH, Lehmann S, Wei YJJ, et al. Association between depression and maintenance medication adherence among Medicare beneficiaries with chronic obstructive pulmonary disease. Int J Geriatr Psychiatry. 2014 [cited 2017 Aug 22]; 29(1): 49-57. DOI: https://doi.org/10.1002/gps.3968.

16. Li J, Fei GH. The unique alterations of hippocampus and cognitive impairment in chronic obstructive pulmonary disease. Respiratory Research. 2013 [cited 2017 Aug 25]; 14:140. DOI: https://doi.org/10.1186/1465-9921-14-140.

17. Neves MCLC, Neves YCS, Mendes CMC, Bastos MN, Camelier AA, Queiroz CF. Evaluation of atopy in patients with COPD. J Bras Pneumol. 2013 [cited 2017 Aug 22]; 39(3): 296-305. DOI: http://dx.doi.org/10.1590/S1806-37132013000300006.

18. Moullec G, Lavoie KL, Rabhi K, Julien M, Favreau H, Labrecque M. Effect of an integrated care programme on re-hospitalization of patients with chronic obstructive pulmonary disease. Respirology. 2012 [cited 2017 Aug 22]; 17(4): 707-14. DOI: https://doi.org/10.1111/j.1440-1843.2012.02168.x.

19. Fonseca PB, Fernández JL, España FV, Ruiz AG, Torres DP, Fernández FL. Is it possible to diagnose the therapeutic adherence of patients with COPD in clinical practice? A cohort study. BMC BMC Pulm Med. 2011 [cited 2017 Aug 25]; 11: 6. DOI: https://doi.org/10.1186/1471-2466-11-6.

20. Liang CY, Chen YJ, Sheu SM, Tsai CF, Chen W. Misuse of inhalers among COPD patients in a community hospital in Taiwan. Int J Chron Obstruct Pulmon Dis. 2018 [cited 2018 Nov 27]; 13: 1309-16. DOI: https://doi.org/10.2147/COPD.S158864.

21. Molimard M, Raherison C, Lignot S, Balestra A, Lamarque S, Chartier A, et al. Chronic obstructive pulmonary disease exacerbation and inhaler device handling: real-life assessment of 2935 patients. Eur Respir J. 2017 [cited 2017 Aug 24]; 49 : 1601794. DOI: https://doi.org/10.1183/13993003.01794-2016.

22. Hernandes NA, Teixeira DC, Probst VS, Brunetto AF, Ramos EMC, Pitta F. Profile of the level of physical activity in the daily lives of patients with COPD in Brazil. J Bras Pneumol. 2009 [cited 2017 Aug 22]; 35(10): 949-56. DOI: https://doi.org/10.1590/s180637132009001000002.

23. Costa GMO, Santos RCAS, Marinho PEM. Daily life activity and depressive symptoms in patients with chronic obstructive pulmonary disease. ConScientiae Saúde. 2017 [cited 2017 Aug 25]; 16(4): 402-408. DOI: https://doi.org/10.5585/conssaude.v16n4.7662.

24. Li Y, Feng J, Li Y, Jia W, Qian H. Assessment of a Domiciliary Integrated Pulmonary Rehabilitation Program for Patients with a History of Acute Exacerbation of Chronic Obstructive Pulmonary Disease: A Retrospective 12-Month Observational Study. Med Sci Monit. 2018 [cited 2018 Nov 278]; 24: 5054-63. DOI: https://doi.org/10.12659/MSM.908463.

25. Burgel PR, Meyer PN, Chanez P, Caillaud D, Carre' P, Perez T, et al. Cough and sputum production are associated with frequent exacerbations and hospitalizations in COPD subjects. Chest. 2009 [cited 2017 Aug 25]; 135(4): 975-82. DOI: https://doi.org/10.1378/chest.08-2062.

26. Troosters T, Molen T, Polkey M, Rabinovich RA, Vogiatzis I, Weisman I, et al. Improving physical activity in COPD: towards a new paradigm. Respir Res. 2013 [cited 2017 Aug 25]; 14:115. DOI: https://doi.org/10.1186/1465-9921-14-115.

27. Brandt CL. Study of older adults' use of self-regulation for COPD self-management informs an evidence-based patient teaching plan. Rehabil Nurs. 2013 [cited 2017 Aug 22]; 38(1): 11-23. DOI: https://doi.org/10.1002/rnj.56.

28. Almagro P, Castro A. Helping COPD patients change health behavior in order to improve their quality of life. Int J Chron Obstruct Pulmon Dis. 2013 [cited 2017 Jul 30]; 8: 335-345. DOI: https://doi.org/10.2147/COPD.S34211.

29. López-López L, Torres-Sánchez I, González-Jiménez E, Díaz-Pelegrina A, Merlos-Navarro S, Valenza MC. Enfermedad pulmonar obstructiva crónica severa y malnutrición: efecto sobre la sintomatología y la función. Nutr Hosp. 2016 [cited 2017 Aug 25]; 33: 319-323. DOI: http://dx.doi.org/10.20960/nh.111.

30. Taddeo OS, Gomes KWL, Caprara A, Gomes AMA, Oliveira GC, Moreira TMM. Access, educational practice and empowerment of patients with chronic diseases. Ciênc Saúde Coletiva. 2012 [cited 2017 Aug 22]; 17(11): 2923-30. DOI: http://dx.doi.org/10.1590/S1413-81232012001100009.

31. Collinsworth AW, Brown RM, James CS, Stanford RH, Alemayehu D, Priest EL. The impact of patient education and shared decision making on hospital readmissions for COPD. Int J Chron Obstruct Pulmon Dis. 2018 [cited 2018 Nov 27]; 13: 1325-32. DOI: https://doi.org/10.2147/COPD.S154414.

32. Bailey J, Dijkers MP, Gassaway J, Thomas J, Lingefelt P, Kreider SE, et al. Relationship of nursing education and care management inpatient rehabilitation interventions and patient characteristics to outcomes following spinal cord injury: the SCIRehab project. J Spinal Cord Med. 2012 [cited 2017 Aug 22]; 35(6): 593-610. DOI: http://dx.doi.org/10.1179/2045772312Y.0000000067 